\title{
Diffuse cavernous hemangioma of the skull misdiagnosed as skull metastasis in breast cancer patient: one case report and literature review
}

\author{
Huizhi Liu ${ }^{1+}$, Xiaojing Chang ${ }^{1 \dagger}$, Hua Shang ${ }^{2}$, Feng Li ${ }^{1}$, Huandi Zhou ${ }^{1}$ and Xiaoying Xue ${ }^{1 *}$
}

\begin{abstract}
Background: Primary intraosseous cavernous hemangiomas (PICHs) of the skull are extremely rare. To date, diffuse cranial hemangioma of skull has not been reported. In cancer patients, it is often misdiagnosed as metastasis.

Case presentation: Here, we presented a case of a 50-year-old female patient suffering from slightly headache who received breast cancer modified radical mastectomy in 2004, computed tomography and magnetic resonance imaging findings revealed abnormal lesions of diffuse skull which were misdiagnosed as skull metastasis, and the relevant literatures were also reviewed.

Conclusions: Diffuse cavernous hemangioma of the skull is exceedingly rare, and imaging data are not typical. The condition is often misdiagnosed, and pathological evaluation is necessary and important. In cases where the mass cannot be completely removed by surgery, radiotherapy could be beneficial.
\end{abstract}

Keywords: Cavernous hemangioma of skull, Metastasis, Misdiagnosis, Breast cancer

\section{Background}

Primary intraosseous cavernous hemangiomas (PICHs) are benign vascular tumors that are characterized by osteolytic bone destruction and often occur in the vertebral column but are extremely rare in the skull [1]. To date, there have been fewer than 100 reported cases of skull PICHs, and the reports were mainly concentrated in Europe, North America, and East Asia [2]. Diffuse cranial hemangioma has not yet been reported. In cancer patients, this condition is often misdiagnosed as metastasis. Here, we reported a case of hemangioma of the skull in a breast cancer patient, which was misdiagnosed as skull metastasis and a review of the literature.

\section{Case presentation}

The patient was a 50-year-old female who underwent surgery in 2004 for mucinous breast carcinoma of the right

*Correspondence: xxy6412@163.com; xxy0636@163.com

Huizhi Liu and Xiaojing Chang have contributed equally to this manuscript.

${ }^{1}$ Department of Radiotherapy, the Second Hospital of Hebei Medical

University, Shijiazhuang 050000, China

Full list of author information is available at the end of the article breast [T2N1M0 (IIb)], and received postoperative CMF (cyclophosphamide+methotrexate+fluorouracil) chemotherapy, radiotherapy with 50Gy and endocrinotherapy with tamoxifen for 5 years. Unfortunately, she did not receive any follow-up examination after completion of the treatment. In 2015, she found that the right local surface of the scalp had become irregular and was increasing in size without pain or numbness. More than 1 year later, the patient experienced a slight headache which was relieved with antipyretic analgesics. Then edema of the frontal scalp and bilateral upper eyelid followed, particularly on the right side. She denied any past trauma history. The physical examination revealed the following: edema of the bilateral frontal scalp and upper eyelid, the right frontal, temporal, and dorsal scalp were slightly lumpy with normal scalp color, and the lumps were immobile and solid but without tenderness. The right thoracic wall was modified due to the prior radical mastectomy for the treatment of breast cancer and the right upper limb was free of edema. Neurological examination revealed no abnormalities other than the slight headache. 
No abnormalies were found in the remaining examinations. Laboratory examination: The results of routine blood and urine examinations were normal, as were those of the biochemistry examination.

Computed tomography $(\mathrm{CT})$ revealed that the bilateral frontal bone, right temporal bone and right parietal bone were diffusely and osteolytically destroyed with soft tissue lesions. (Fig. 1). No metastatic lesions were observed on the CT images of the chest, abdomen, and pelvis. The magnetic resonance imaging (MRI) results revealed that the bilateral frontal bone, right temporal bone, and right parietal bone were thickened with nodules. The lesions were tent-like on coronal and sagittal planes (Fig. 2e, f). The lesions exhibited a slight hypointensity on T1-weighted imaging (T1WI) (Fig. 2a), and isointensity on T1-weighted imaging (T2WI) (Fig. 2b) and T2-fluid-attenuated inversion recovery (FLAIR) (Fig. 2c) images, and were significantly enhanced (Fig. 2d, e, f) after enhancement. The meninges of the right frontal, temporal, and parietal areas were also diffusely thickened with nodules. The nodules compressed the right temporal lobe slightly and led to edema of the parenchyma (Fig. 2a, b, c), which was not enhanced (Fig. 2d, e). There was slight compression of the adjacent brain parenc hyma and ventricle. The brain midline structure shifted to the left by approximately $0.5 \mathrm{~cm}$, and the size of the meningeal nodular lesions was approximately $2.8 \times 1.3 \times 2.8 \mathrm{~cm}$. The tumor marker levels were: carcinoembryonic antigen
(CEA) $11.52 \mathrm{ng} / \mathrm{ml}$, cancer antigen 15-3(CA15-3) $77.39 \mathrm{U} /$ $\mathrm{ml}$. The results combined with the medical history, led us to suspect the presence of a metastatic tumor with invasion of the right temporal and occipital meninges. Systemic bone imaging revealed abnormal bone metabolism in the skull (Fig. 3). To evaluate the nature of the lesion, open biopsy was performed. The pathological diagnosis of the biopsy was hemangioma of the skull (Fig. 4). The corrected diagnosis was cranial cavernous hemangioma.

Based on the CT and MRI findings, the headache and upper eyelid edema of the patient were considered to be caused by the local invasion of the skull lesions. The patient received a normal three-dimensional conformal radiotherapy with a dose of $60 \mathrm{~Gy} / 30 \mathrm{~F}$ (the lesions nearby bilateral upper orbital bone was 50Gy/30F). The target volume was described as: according to $\mathrm{CT}$ and MRI images to delineate the target, gross tumor volume (GTV) comprised the skull, meningeal and Right temporal lobe lesions, GTV was enlarged $3 \mathrm{~mm}$ circumferentialy to form the planning target volume (PTV). Irradiation was performed using $6 \mathrm{MV} \mathrm{X}$-rays. At the end of the radiotherapy, the size of the lump at biopsy site (the primary right frontal, temporal, and top scalp) was slightly reduced on physical examination, but the head CT showed no significantly change compared to pre-radiotherapy CT images (Fig. 5). The symptoms of discomfort in the head and eye swelling were relieved. We concluded that the blood vessels of lesions in skull may be blocked. The

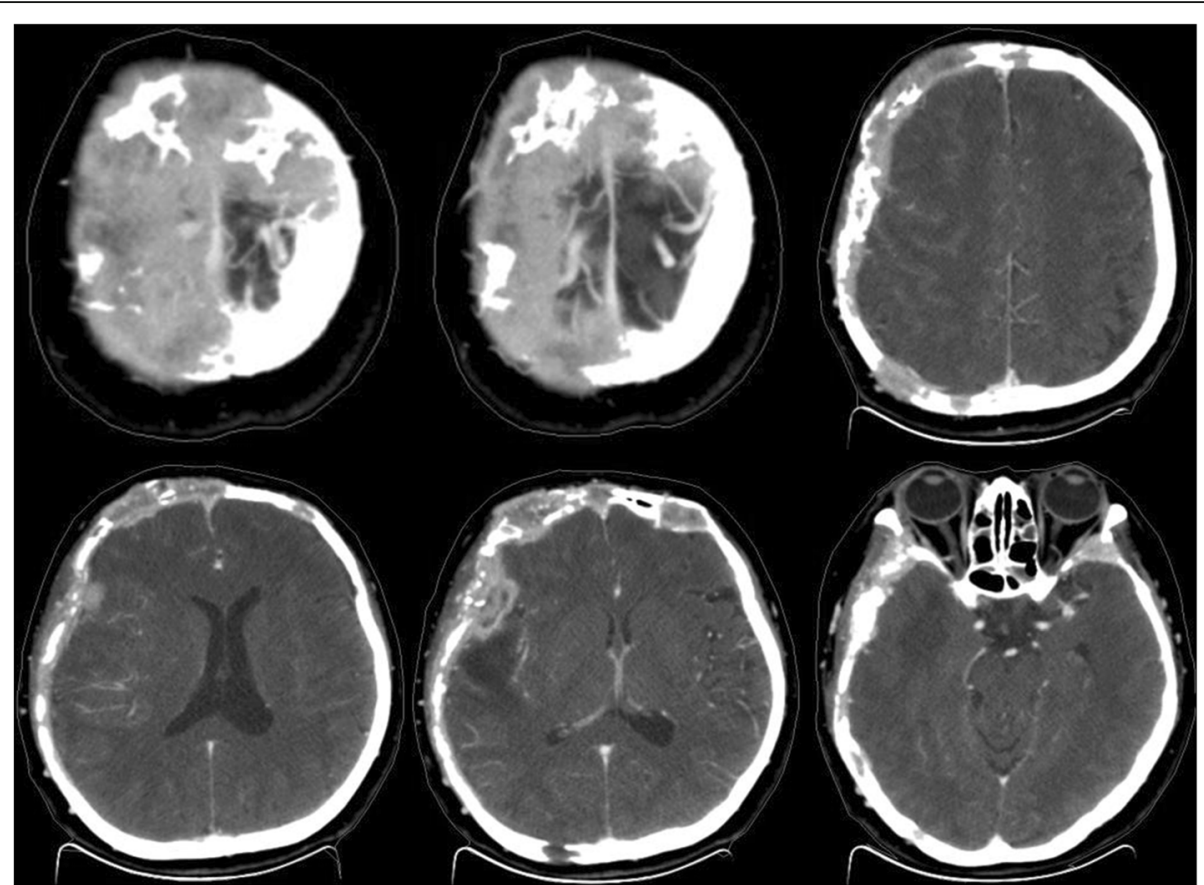

Fig. 1 Computed tomography revealed that the right temporal lobe, and bilateral frontal lobes contained lesions. Meanwhile, the bilateral forehead, and right temporal occipital exhibited diffuse osteolytic bone destruction. The tesions had a "bean curd" type of morphology. The border of the lesion was undefined. The surrounding bone without hardening 


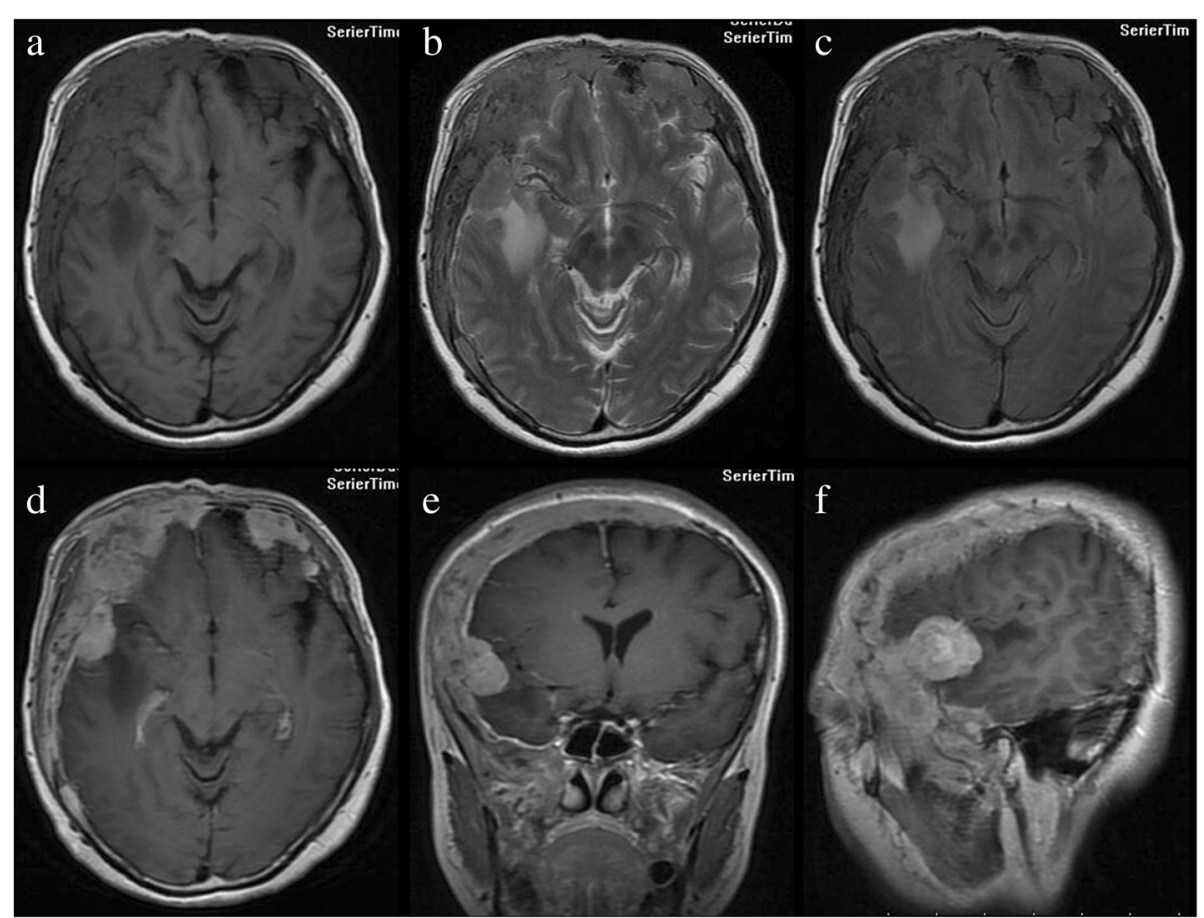

Fig. 2 Magnetic resonance imaging revealed that thickening of the bilateral forehead, right superior temporal occipital bone and bilateral upper orbital bone. There were multiple visible flaps and nodules along with slightly longer T1 (a), slightly longer T2 (b), and other FLAIR (c) signals in these areas of bone thickening. An enhanced scan revealed slightly irregular enhancement $(\mathbf{d}, \mathbf{e}, \mathbf{f})$. Significantly enhanced nodules could be observed on the right side of the temporal and temporal occipital meninges. The largest nodule was at the right side of the temporal lobe, and pressure spread to the adjacent brain parenchyma, around pressure exist the long T1 long T2 edema signal. There was slight compression of the adjacent brain parenchyma and ventricle

patient reported no symptoms during the 1-year telephonic follow-up. Unfortunately, the CT scan during 1-year follow-up was performed at an outside institution and was not available for inclusion in this report.

\section{Discussion and conclusions}

Primary hemangiomas could be divided into three types: cavernous, capillary, and mixed. Cavernous hemangioma is the most common type in the skull, accounting for $10 \%$ of all benign tumors and $0.2 \%$ of cranial tumors of the skull [3]. Hemangiomas could occur in any part of the skull, such as the clivus $[4,5]$, frontal bone $[6,7]$, parietal bone or petrous bone [8], but are predominantly located in the frontal bone, with $44.1 \%$ of skull PICHs reported in this area in previous studies [2]. To date, the origin of hemangiomas remains unclear. Some researchers have proposed that cavernous hemangioma may be an inherited disease, and a mutation in KRIT1/CCM1 gene which encodes a protein that affects endothelial cell structure and function is correlated with the pathogenic mechanisms of cavernous hemangioma [9]. Recent studies have reported that trauma and ionizing radiation particularly treatment with stereotactic radiosurgery (SRS) for brain tumors may be related to the development of cavernous hemangioma [10-12]. It was worth mentioning that our patient received radiotherapy of the right chest wall combined regional $>10$ years prior due to breast cancer. Hemangiomas of the skull primarily present as a single mass, with a few cases of multiple masses [13, 14]. Hemangiomas of the skull typically occur in patients in the fourth and fifth decades of life, and the incidence rate in men and women is approximately 1:2-3 [15]. Hemangiomas of the skull originate from the plate barrier, and spread along both sides of the barrier, which is indicative of an osteolytic mass [2]. Hemangiomas of the skull are predominantly solitary, and rarely invade the entire skull. To our knowledge, we report the first case of a diffuse cavernous hemangioma of the skull, wherein the CT and MRI results lead to the misdiagnosis of skull metastasis. Hemangiomas of the skull have no typical clinical symptoms: some patients present with chronic headaches, while some have no significant complaints aside from a local swelling [16].

Cavernous hemangiomas consist of clusters of dilated blood vessels, which are separated by fibrous septa, whereas capillary ones are rich in small vascular luminas without a significant number of fibrous septa. Radiological evaluation includes standard skull X-rays, CT scans, and MRI. X-ray is the most basic method of examination, while $\mathrm{CT}$ is the most commonly used method of inspection. 


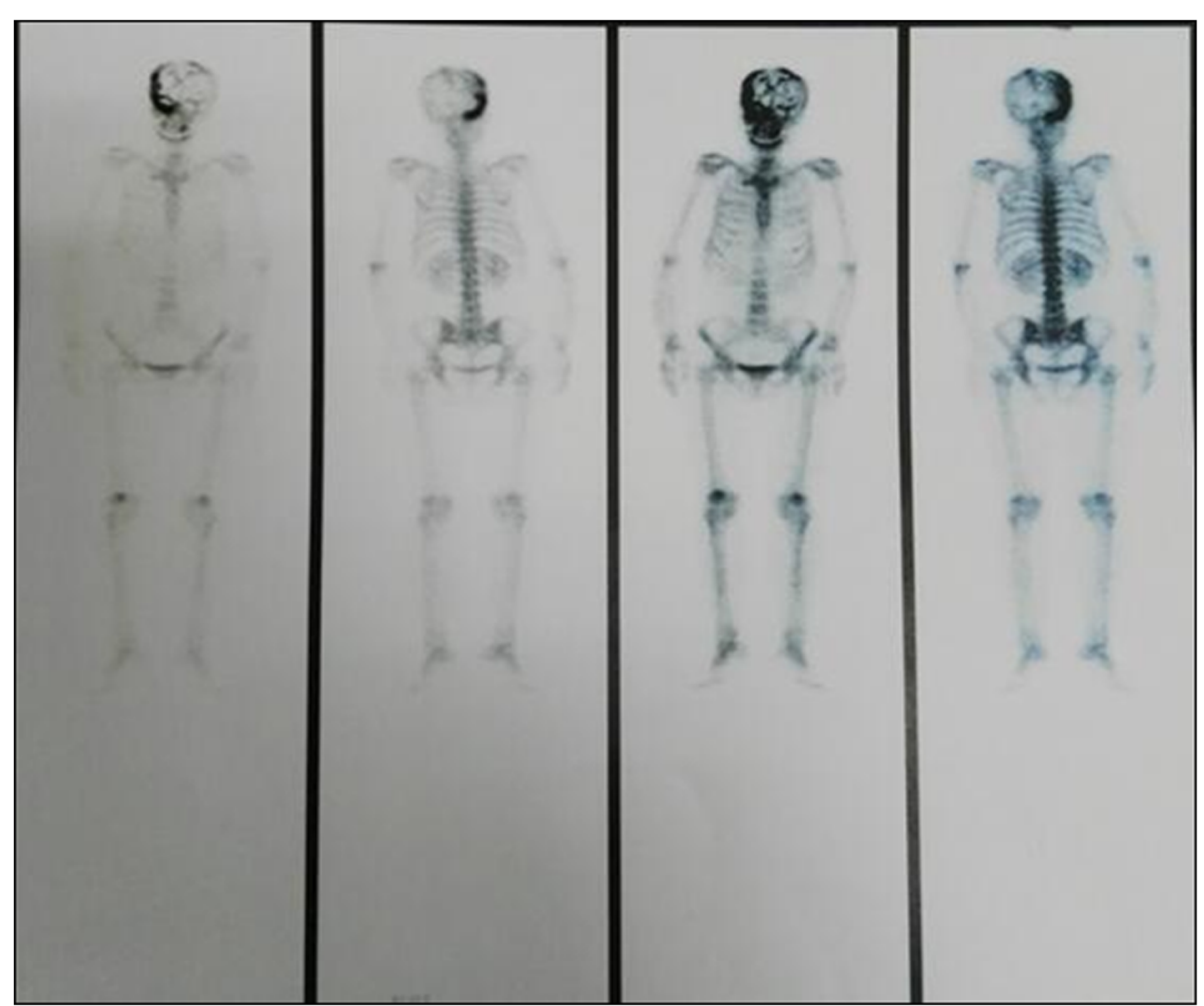

Fig. 3 Systemic bone imaging revealed abnormal bone metabolism in the skull bone

However, PICHs of the skull do not have typical radiological features. Non-enhanced CT typically reveals a mass, which represents an osteolytic lesion, with intense enhancement after intravenous contrast administration. MRI of the lesion was inconclusive on T1-weighted images with isointensity or hyperintensity, and irregular hyperintensity on T2 weighted images [17]. The lesion exhibited intense homogenous postcontrast enhancement.
For a single mass, complete resection was beneficial, and the cosmetic results of most cases were satisfactory and revealed no recurrence within a short period of time $[18,19]$. If the cranial cavernous hemangioma was relatively large, embolization combined with craniotomy may be the superior treatment [20]. However, multiple lesions may require combined radiotherapy, particularly for diffuse cranial hemangiomas. Such as this case, the

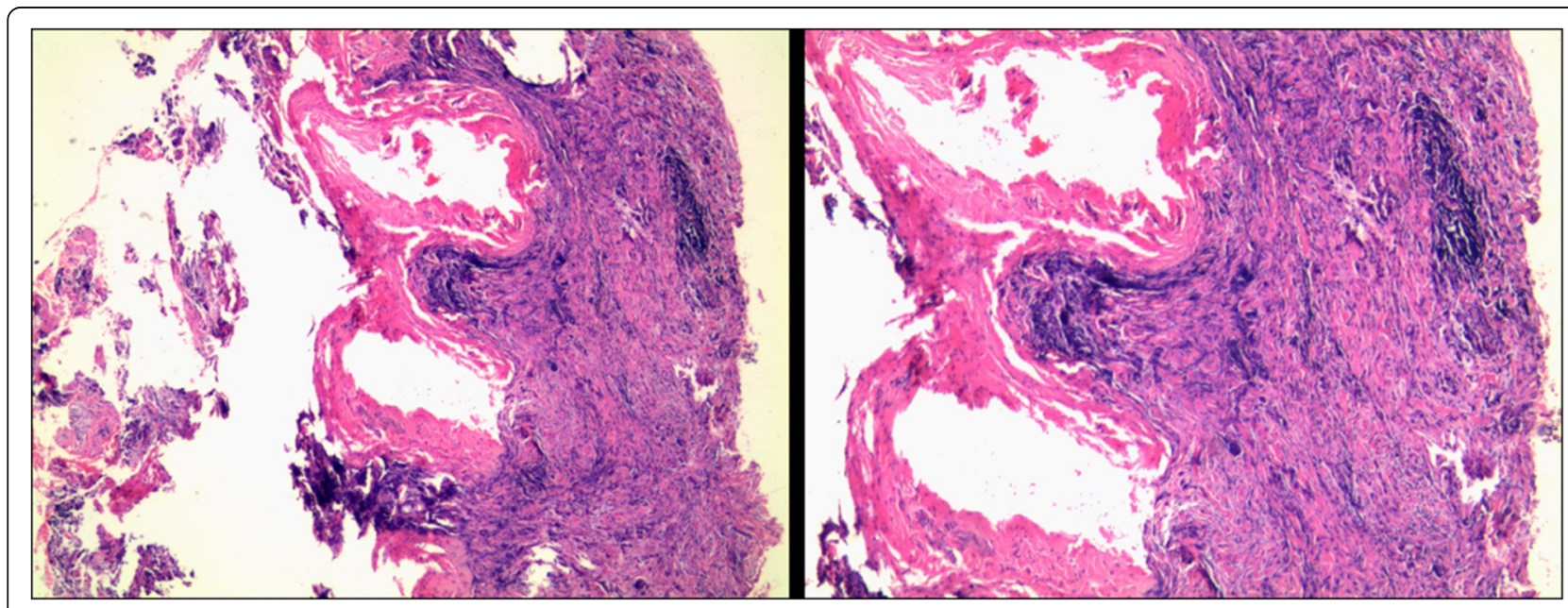

Fig. 4 Pathological analysis of the biopsy revealed a hemangioma 


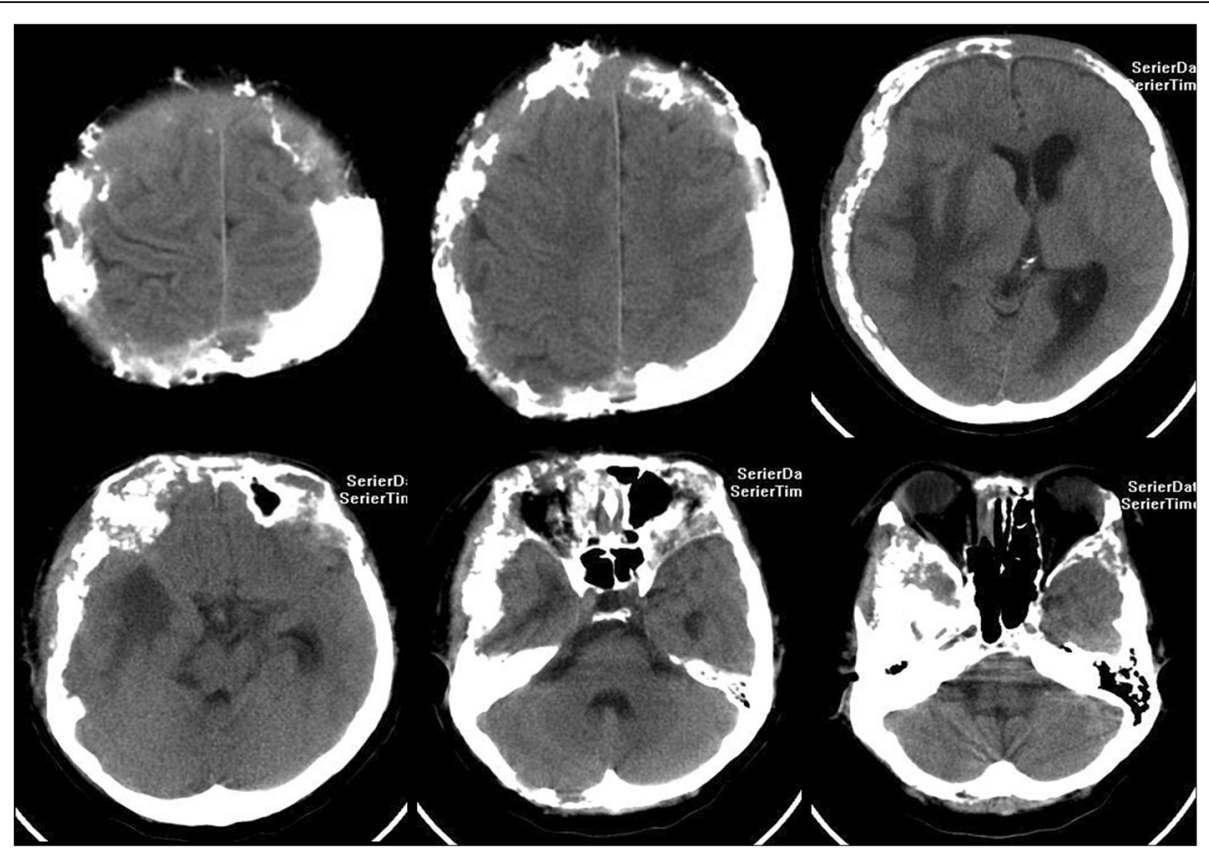

Fig. 5 The head computed tomography scan which was performed th the end of radiation therapy revealed no significant change compared to that before radiotherapy

treatment efficacy of radiotherapy which relieved the discomfort symptoms of the patient was satisfactory, although skull lesions had no significant change in size. Given the condition that long-term radiographic imaging was helpful for assessing the efficacy of radiation therapy for PICHs, the patient was advised to attend regular follow-up assessments. In addition, it is well known that tumors result from the abnormal expression of genes events, and some recent studies have reported that mutation of some genes was related to development of hemangiomas $[21,22]$. If we could perform gene detection in cases of hemangioma of the skull, and then recommend targeted therapy, it could be a prominent topic in future studies.

In this case, the patient received surgery for mucinous breast carcinoma in 2004, and received postoperative chemoradiotherapy, which led to the misdiagnosis of metastasis. In addition, hemangiomas of the skull often grow slowly, over several months to several years, the symptoms are not typical, and often present as a single mass or multiple lesions with osteolytic lesions [13, 14]. In this case, although the intraosseous lesion of the skull presented as an osteolytic lesion, the CT and MRI images indicated that the lesion was diffuse. In addition, some enhanced nodules with variable surrounding vasogenic edema were observed on the right side of the temporal and temporal occipital meninges, which presented occupying lesion making the brain midline structure shift to the left. Furthermore, the CT image revealed diffuse osteolytic bone destruction, which seemed to be a "bean curd" type of change, and the border of the lesion was not clear. All of the above characteristics were similar to those of metastatic lesions [23, 24]. Furthermore, the level of the tumor marker, CA15-3, was higher. It is well-known that more patients with metastatic breast cancer have elevated CA15-3 levels. Based on the above, metastasis of the skull was diagnosed. However, the location of the lesions, which were mainly in the skull, was rare in cases of metastatic lesions. Thus, the scalp thickening lesions were biopsied, and the pathological diagnosis was hemangioma of the skull. The lesion of the cranial hemangioma grew rapidly and the local surface irregularity was apparent. After radiotherapy, the size of the lump at biopsy site was reduced and the distending pain of the head was alleviated. CA15-3 may be considered an early warning sign and was found to be indicative of a high risk of recurrence in breast cancer patients. For this case, CA15-3 may be an early warning sign of recurrence of breast cancer. Therefore, the patient was advised to attend regular follow-up assessments.

In conclusion, $\mathrm{PICH}$ of the skull are rare benign lesions characterized by osteolytic bone destruction and have no typical clinical symptoms. Diffuse cavernous hemangioma of the skull is exceedingly rare, and imaging findings are not specific, which leads to misdiagnosis. Pathological evaluation is therefore necessary and important. In cases wherein the mass could not be completely removed by surgery, and radiotherapy would be beneficial. 


\section{Abbreviations}

CA15-3: Cancer antigen 15-3; CEA: Carcinoembryonic antigen; CMF: Cyclophosphamide+methotrexate+fluorouracil; CT: Computed tomography; FLAIR: Fluid-attenuated inversion recovery; GTV: Gross tumor volume; MRI: Magnetic resonance imaging; PICHs: Primary intraosseous cavernous hemangiomas; PTV: Planning target volume; SRS: Stereotactic radiosurgery; T1Wl: T1-weighted imaging; T2WI: T1-weighted imaging

\section{Acknowledgements \\ Not applicable.}

\section{Funding}

Not applicable.

\section{Availability of data and materials}

All data of CT, MRI, Systemic bone imaging and pathology analyzed during this study are included in this published article.

\section{Authors' contributions}

$\mathrm{HL}$ treated the patient, participated in the study conception, and participated in the acquisition of data. $X C$ participated in the treatment of the patient, analyzed of aggregated data and drafted this manuscript. FL participated in the acquisition of data and histological examination of the sample. HS participated in the radiological examination of the images. $\mathrm{HZ}$ participated in the acquisition of data and revision of the manuscript. XX participated in the critical review and substantively revised it. All the authors have read and approved the final manuscript.

\section{Ethics approval and consent to participate}

This case report was approved by the institutional review board of Hebei Medical University.

\section{Consent for publication}

Written informed consent was obtained from the patient for publication of this case report and any accompanying images.

\section{Competing interests}

The authors declare that they have no competing interests.

\section{Publisher's Note}

Springer Nature remains neutral with regard to jurisdictional claims in published maps and institutional affiliations.

\section{Author details}

${ }^{1}$ Department of Radiotherapy, the Second Hospital of Hebei Medical University, Shijiazhuang 050000, China. ${ }^{2}$ Department of medical imaging, the Second Hospital of Hebei Medical University, Shijiazhuang, China.

Received: 14 January 2018 Accepted: 1 February 2019

Published online: 25 February 2019

\section{References}

1. Naama O, Gazzaz M, Akhaddar A, Belhachmi A, Asri A, Elmostarchid B, Elbouzidi A, Kadiri B, Boucetta M. Cavernous hemangioma of the skull: 3 case reports. Surg Neurol. 2008;70:654-9.

2. Yang Y, Guan J, Ma W, Li Y, Xing B, Ren Z, Su C, Wang R. Primary intraosseous cavernous hemangioma in the skull. Medicine (Baltimore). 2016;95(11):e3069

3. Rumana M, Khursheed N, Farhat M, Othman S, Masood L. Congenital intraosseous cavernous hemangioma of the skull: an unusual case. Pediatr Neurosurg. 2013:49(4):229-31.

4. Gologorsky Y, Shrivastava RK, Panov F, Mascitelli J, Signore AD, Govindaraj S, Smethurst M, Bronster DJ. Primary intraosseous cavernous hemangioma of the clivus: case report and review of the literature. J Neurol Surg Rep. 2013;74(1):17-22.

5. Serrano L, Archavlis E, Januschek E, Ulrich PT. High risk of cerebrospinal fluid leakage in surgery of a rare primary intraosseous cavernous hemangioma of the clivus showing meningeal infiltration: a case report and review of the literature. Surg Neurol Int. 2015;6(Suppl 3):S117-23.

6. Park BH, Hwang E, Kim CH. Primary intraosseous hemangioma in the frontal bone. Arch Plast Surg. 2013;40(3):283-5.
7. Murrone D, De Paulis D, Millimaggi DF, Del Maestro M, Galzio RJ. Cavernous hemangioma of the frontal bone: a case report. J Med Case Rep. 2014;8:121.

8. Sade B, Lee DK, Prayson RA, Hughes GB, Lee JH. Intraosseous cavernous angioma of the petrous bone. Skull Base. 2009:19(3):237-40.

9. Reddy S, Gorin MB, McCannel TA, Tsui I, Straatsma BR. Novel KRIT1/ CCM1 mutation in a patient with retinal cavernous hemangioma and cerebral cavernous malformation. Graefes Arch Clin Exp Ophthalmol. 2010;248(9):1359-61.

10. Haeren RH, Dings J, Hoeberigs MC, Riedl RG, Rijkers K. Posttraumatic skull hemangioma: case report. J Neurosurg. 2012;117(6):1082-8.

11. Yu J, Li Y, Duan X. Posttraumatic cavernous hemangioma of the skull. J Craniofac Surg. 2014;25(1):e48-51.

12. Cha YJ, Nahm JH, Ko JE, Shin HJ, Chang J-H, Cho NH, Kim SH. Pathological evaluation of radiation-induced vascular lesions of the brain: distinct from de novo cavernous hemangioma. Yonsei Med J. 2015;56(6):1714-20.

13. Tyagi DK, Balasubramaniam S, Sawant HV. Giant primary ossified cavernous hemangioma of the skull in an adult: a rare calvarial tumor. J Neurosci Rural Pract. 2011;2(2):174-7.

14. Xu P, Lan S, Liang Y, Xiao Q. Multiple cavernous hemangiomas of the skull with dural tail sign: a case report and literature review. BMC Neurol. 2013;13:155.

15. Salunke P, Sinha R, Khandelwal NK, Kumar A, Gupta K, Mukherjee KK. Primary intraosseus cavernous hemangioma of the skull base. $\mathrm{Br}$ Neurosurg. 2010;24:84-5.

16. Liu JK, Burger PC, Harnsberger HR, Couldwell WT. Primary intraosseous skull base cavernous hemangioma: case report. Skull Base. 2003;13(4):219-28.

17. Kilani M, Darmoul M, Hammedi F, Ben Nsir A, Hattab MN. Cavernous hemangioma of the skull and meningioma: association or coincidence? Case Rep Neurol Med. 2015;2015:716837.

18. Clauser L, Mandrioli S, Polito J, Galiè M. Surgical techniques for the removal of forehead hemangioma. J Craniofac Surg. 2006;17(4):702-4.

19. Dogan S, Kocaeli H, Sahin S, Korfali E, Saraydaroglu O. Large cavernous hemangioma of the frontal bone. Neurol Med Chir (Tokyo). 2005;45(5):264-7.

20. Nasrallah IM, Hayek R, Duhaime AC, Stotland MA, Mamourian AC. Cavernous hemangioma of the skull: surgical treatment without craniectomy. J Neurosurg Pediatr. 2009:4(6):575-9.

21. Lee DM, Yu SH, Yoon HH, Lee KL, Eom YS, Lee K, Kim B-J, Kim YS, Park IB, Kim K-W, Lee S. Genetic analysis of multiple endocrine neoplasia type 1 (MEN1) leads to misdiagnosis of an extremely rare presentation of intrasellar cavernous hemangioma as MEN1. Endocrinol Metab (Seoul). 2014;29(2):146-53.

22. Lanfranconi S, Ronchi D, Ahmed N, Civelli V, Basilico P, Bresolin N, Comi GP, Corti S. A novel CCM1 mutation associated with multiple cerebral and vertebral cavernous malformations. BMC Neurol. 2014;14:158.

23. Fink KR, Fink JR. Imaging of brain metastases. Surg Neurol Int. 2013; 4(Suppl 4):S209-19.

24. Villanueva-Meyer JE, Mabray MC, Cha S. Current clinical brain tumor imaging. Neurosurgery. 2017:81(3):397-415.

\section{Ready to submit your research? Choose BMC and benefit from:}

- fast, convenient online submission

- thorough peer review by experienced researchers in your field

- rapid publication on acceptance

- support for research data, including large and complex data types

- gold Open Access which fosters wider collaboration and increased citations

- maximum visibility for your research: over $100 \mathrm{M}$ website views per year

At BMC, research is always in progress.

Learn more biomedcentral.com/submissions 